TAIWANESE JOURNAL OF MATHEMATICS

Vol. 19, No. 1, pp. 133-157, February 2015

DOI: $10.11650 /$ tjm.19.2015.4504

This paper is available online at http://journal.taiwanmathsoc.org.tw

\title{
REFINEMENTS OF HERMITE-HADAMARD TYPE INEQUALITIES FOR DIFFERENTIABLE CO-ORDINATED CONVEX FUNCTIONS AND APPLICATIONS
}

\author{
Kai-Chen Hsu
}

\begin{abstract}
In this paper, we shall establish some inequalities for differentiable co-ordinated convex and concave functions on a rectangle from the plane. They are connected with the left side of extended Hermite-Hadamard inequality in two variables. Also, these inequalities are able to be applied to some special means and cubature formulae.
\end{abstract}

\section{INTRODUCTION}

Throughout this paper, let $\Delta:=[a, b] \times[c, d]$ be double intervals with $a<b, c<d$ in $\mathbb{R}^{2}$, and a partial derivative of second order $\frac{\partial^{2} f}{\partial y \partial x}$ is denoted by $f_{x y}$ for brevity.

The inequality

$$
f\left(\frac{a+b}{2}\right) \leq \frac{1}{b-a} \int_{a}^{b} f(t) d t \leq \frac{f(a)+f(b)}{2},
$$

which holds for all convex functions $f:[a, b] \rightarrow \mathbb{R}$ is known as Hermite-Hadamard's inequality [5] or simply Hadamard's inequality.

For some results which generalize, improve, and extend the inequality (1.1), please refer to [1-4] and [6-16].

Let us consider a function $f(x, y)$ on a convex subset $\Delta$ on $\mathbb{R}^{2}$. Recall that if the function $f(x, y)$ defined on $\Delta$ is convex and any non-negative constant $\alpha \in[0,1]$ and any two points $X, Y \in \Delta$ are both satisfied, the following inequality holds:

$$
f(\alpha X+(1-\alpha) Y) \leq \alpha f(X)+(1-\alpha) f(Y) .
$$

Based on the above inequality, Dragomir proposed the concept of co-ordinated convex functions in [2], defined as follows:

Received March 8, 2014, accepted May 7, 2014.

Communicated by Jen-Chih Yao.

2010 Mathematics Subject Classification: Primary 26D15; Secondary 26A51.

Key words and phrases: Hermite-Hadamard's inequality, Convex function, Co-ordintaed convex function, Hölder's inequality, Jensen's integral inequality. 
Definition 1. A function $f: \Delta \rightarrow \mathbb{R}$ is said to be convex on the co-ordinates on $\Delta$ if the partial mappings

$$
\left\{\begin{aligned}
f(u, y):[a, b] & \rightarrow \mathbb{R}, \quad \forall y \in[c, d] \\
f(x, v):[c, d] \rightarrow \mathbb{R}, & \forall x \in[a, b]
\end{aligned}\right.
$$

are convex.

Definition 2. A function $f: \Delta \rightarrow \mathbb{R}$ is said to be convex on the co-ordinates on $\Delta$ if the inequality

$$
\begin{aligned}
& f(t x+(1-t) y, s u+(1-s) w) \\
\leq & t s f(x, u)+t(1-s) f(x, w)+s(1-t) f(y, u)+(1-t)(1-s) f(y, w),
\end{aligned}
$$

holds for all $t, s \in[0,1]$ and $(x, u),(x, w),(y, u),(y, w) \in \Delta$.

Clearly, we can observe that every convex function $f: \Delta \rightarrow \mathbb{R}$ is convex on the co-ordinates, but in some special cases, some co-ordinated convex functions are not convex (please refer to [2]). For more relevant co-ordinated convex functions, please refer to [4], [7-9] and [11].

The following extended Hadamard's inequality for co-ordinated convex functions on $\Delta$ in two variables was proved in [2]:

Theorem A. Suppose that $f: \Delta \rightarrow \mathbb{R}$ is co-ordinated convex on $\Delta$. Then the following inequalities hold:

$$
\begin{aligned}
& f\left(\frac{a+b}{2}, \frac{c+d}{2}\right) \\
\leq & \frac{1}{2}\left[\frac{1}{b-a} \int_{a}^{b} f\left(x, \frac{c+d}{2}\right) d x+\frac{1}{d-c} \int_{c}^{d} f\left(\frac{a+b}{2}, y\right) d y\right] \\
\leq & \frac{1}{(b-a)(d-c)} \int_{c}^{d} \int_{a}^{b} f(x, y) d x d y \\
\leq & \frac{1}{4}\left[\frac{1}{b-a} \int_{a}^{b} f(x, c) d x+\frac{1}{b-a} \int_{a}^{b} f(x, d) d x\right. \\
& \left.+\frac{1}{d-c} \int_{c}^{d} f(a, y) d y+\frac{1}{d-c} \int_{c}^{d} f(b, y) d y\right] \\
\leq & \frac{f(a, c)+f(a, d)+f(b, c)+f(b, d)}{4} .
\end{aligned}
$$

The above inequalities are sharp.

In [9], Latif and Dragomir established the following Hadamard type inequalities that gave an estimate of the difference in the inequalities (1.3) for differentiable coordinated convex functions on $\Delta$. 
Theorem B. Let $f: \Delta \rightarrow \mathbb{R}$ be a partial differentiable mapping on $\Delta$. If $\left|f_{x y}\right|$ is convex on the co-ordinates on $\Delta$, then the following inequality holds:

$$
\begin{aligned}
& \left|\frac{1}{(b-a)(d-c)} \int_{a}^{b} \int_{c}^{d} f(x, y) d y d x+f\left(\frac{a+b}{2}, \frac{c+d}{2}\right)-P\right| \\
\leq & \frac{(b-a)(d-c)}{16}\left[\frac{\left|f_{x y}(a, c)\right|+\left|f_{x y}(a, d)\right|+\left|f_{x y}(b, c)\right|+\left|f_{x y}(b, d)\right|}{4}\right],
\end{aligned}
$$

where

$$
P=\frac{1}{b-a} \int_{a}^{b} f\left(x, \frac{c+d}{2}\right) d x+\frac{1}{d-c} \int_{c}^{d} f\left(\frac{a+b}{2}, y\right) d y .
$$

Theorem C. Let $f: \Delta \rightarrow \mathbb{R}$ be a partial differentiable mapping on $\Delta$. If $\left|f_{x y}\right|^{q}$ is convex on the co-ordinates on $\Delta$ and $p, q>1, \frac{1}{p}+\frac{1}{q}=1$, then the following inequality holds:

$$
\begin{aligned}
& \left|\frac{1}{(b-a)(d-c)} \int_{a}^{b} \int_{c}^{d} f(x, y) d y d x+f\left(\frac{a+b}{2}, \frac{c+d}{2}\right)-P\right| \\
\leq & \frac{(b-a)(d-c)}{4(p+1)^{\frac{2}{p}}}\left[\frac{\left|f_{x y}(a, c)\right|^{q}+\left|f_{x y}(a, d)\right|^{q}+\left|f_{x y}(b, c)\right|^{q}+\left|f_{x y}(b, d)\right|^{q}}{4}\right]^{\frac{1}{q}},
\end{aligned}
$$

where $P$ is as given in Theorem $B$.

The following theorem shows the result of giving Theorem $\mathrm{C}$ an improved and simplified constant.

Theorem D. Let $f: \Delta \rightarrow \mathbb{R}$ be a partial differentiable mapping on $\Delta$. If $\left|f_{x y}\right|^{q}$ is convex on the co-ordinates on $\Delta$ and $q \geq 1$, then the following inequality holds:

$$
\begin{aligned}
& \left|\frac{1}{(b-a)(d-c)} \int_{a}^{b} \int_{c}^{d} f(x, y) d y d x+f\left(\frac{a+b}{2}, \frac{c+d}{2}\right)-P\right| \\
\leq & \frac{(b-a)(d-c)}{16}\left[\frac{\left|f_{x y}(a, c)\right|^{q}+\left|f_{x y}(a, d)\right|^{q}+\left|f_{x y}(b, c)\right|^{q}+\left|f_{x y}(b, d)\right|^{q}}{4}\right]^{\frac{1}{q}},
\end{aligned}
$$

where $P$ is as given in Theorem $B$.

The following theorem discusses about concave functions on the co-ordinates on $\Delta$.

Theorem E. Let $f: \Delta \rightarrow \mathbb{R}$ be a partial differentiable mapping on $\Delta$. If $\left|f_{x y}\right|^{q}$ is concave on the co-ordinates on $\Delta$ and $q \geq 1$, then the following inequality holds: 


$$
\begin{aligned}
&\left|\frac{1}{(b-a)(d-c)} \int_{a}^{b} \int_{c}^{d} f(x, y) d y d x+f\left(\frac{a+b}{2}, \frac{c+d}{2}\right)-P\right| \\
& \leq \frac{(b-a)(d-c)}{64}\left[\left|f_{x y}\left(\frac{a+2 b}{3}, \frac{c+2 d}{3}\right)\right|+\left|f_{x y}\left(\frac{a+2 b}{3}, \frac{2 c+d}{3}\right)\right|\right. \\
&\left.\quad+\left|f_{x y}\left(\frac{2 a+b}{3}, \frac{c+2 d}{3}\right)\right|+\left|f_{x y}\left(\frac{2 a+b}{3}, \frac{2 c+d}{3}\right)\right|\right],
\end{aligned}
$$

where $P$ is as given in Theorem $B$.

The aim of this paper is to establish some extended Hadamard type inequalities which could improve the Theorems B-D and generalize the Theorems E. Also, the paper aims to note some consequent applications to special means and to provide the cubature formula.

In order to show our main results, we need the following identities (I)-(III):

(I) For $a \leq A \leq b, c \leq C \leq d$, the following four identities hold:

$$
\begin{aligned}
& \int_{c}^{C} \int_{a}^{A}(x-a)(y-c)(A-x)(C-y) d x d y=\frac{1}{36}(A-a)^{3}(C-c)^{3}, \\
& \int_{c}^{C} \int_{a}^{A}(x-a)(y-c)(x-a)(C-y) d x d y=\frac{1}{18}(A-a)^{3}(C-c)^{3}, \\
& \int_{c}^{C} \int_{a}^{A}(x-a)(y-c)(A-x)(y-c) d x d y=\frac{1}{18}(A-a)^{3}(C-c)^{3}, \\
& \int_{c}^{C} \int_{a}^{A}(x-a)(y-c)(x-a)(y-c) d x d y=\frac{1}{9}(A-a)^{3}(C-c)^{3} .
\end{aligned}
$$

(II) For $a \leq A \leq b, a \leq B \leq b, c \leq C \leq d$, the following eight identities hold:

$$
\begin{aligned}
& \int_{C}^{d} \int_{a}^{B}(x-a)(d-y)(B-x)(d-y) d x d y=\frac{1}{18}(B-a)^{3}(d-C)^{3}, \\
& \int_{C}^{d} \int_{a}^{B}(x-a)(d-y)(x-a)(d-y) d x d y=\frac{1}{9}(B-a)^{3}(d-C)^{3}, \\
& \int_{C}^{d} \int_{a}^{B}(x-a)(d-y)(B-x)(y-C) d x d y=\frac{1}{36}(B-a)^{3}(d-C)^{3}, \\
& \int_{C}^{d} \int_{a}^{B}(x-a)(d-y)(x-a)(y-C) d x d y=\frac{1}{18}(B-a)^{3}(d-C)^{3},
\end{aligned}
$$




$$
\begin{aligned}
& \int_{c}^{C} \int_{A}^{b}(b-x)(y-c)(b-x)(C-y) d x d y=\frac{1}{18}(b-A)^{3}(C-c)^{3}, \\
& \int_{c}^{C} \int_{A}^{b}(b-x)(y-c)(x-A)(C-y) d x d y=\frac{1}{36}(b-A)^{3}(C-c)^{3}, \\
& \int_{c}^{C} \int_{A}^{b}(b-x)(y-c)(b-x)(y-c) d x d y=\frac{1}{9}(b-A)^{3}(C-c)^{3}, \\
& \int_{c}^{C} \int_{A}^{b}(b-x)(y-c)(x-A)(y-c) d x d y=\frac{1}{18}(b-A)^{3}(C-c)^{3} .
\end{aligned}
$$

(III) For $a \leq B \leq b, c \leq C \leq d$, the following four identities hold:

$$
\begin{aligned}
& \int_{C}^{d} \int_{B}^{b}(b-x)(d-y)(b-x)(d-y) d x d y=\frac{1}{9}(B-b)^{3}(C-d)^{3}, \\
& \int_{C}^{d} \int_{B}^{b}(b-x)(d-y)(x-B)(d-y) d x d y=\frac{1}{18}(b-B)^{3}(d-C)^{3}, \\
& \int_{C}^{d} \int_{B}^{b}(b-x)(d-y)(b-x)(y-C) d x d y=\frac{1}{18}(b-B)^{3}(d-C)^{3}, \\
& \int_{C}^{d} \int_{B}^{b}(b-x)(d-y)(x-B)(y-C) d x d y=\frac{1}{36}(b-B)^{3}(d-C)^{3} .
\end{aligned}
$$

\section{MAIN Results}

In this section, let the mapping $w_{1}(x, y)$ for all $(x, y) \in \Delta$ be defined as follows:

$$
w_{1}(x, y):= \begin{cases}(x-a)(y-c), & (x, y) \in[a, A] \times[c, C] \\ (x-a)(y-d), & (x, y) \in[a, B] \times(C, d] \\ (x-b)(y-c), & (x, y) \in(A, b] \times[c, C] \\ (x-b)(y-d), & (x, y) \in(B, b] \times(C, d]\end{cases}
$$

Now, we are ready to state and prove the main results.

Theorem 1. Let $f: \Delta \rightarrow \mathbb{R}$ be a partial differentiable mapping on $\Delta$. If $\left|f_{x y}\right|$ is convex on the co-ordinates on $\Delta$, then the following inequality holds:

$$
\begin{aligned}
& \left|\frac{1}{(b-a)(d-c)} \int_{c}^{d} \int_{a}^{b} f(x, y) d x d y+M(A, B ; C)-N(A, B ; C)\right| \\
\leq & E(A, B ; C)+R(A, B ; C)
\end{aligned}
$$


where

$$
\begin{aligned}
& M(A, B ; C):=\frac{C-c}{d-c} f(A, C)+\frac{d-C}{d-c} f(B, C) \\
& N(A, B ; C):= \frac{1}{b-a} \int_{a}^{b} f(x, C) d x+\frac{1}{d-c}\left[\int_{c}^{C} f(A, y) d y+\int_{C}^{d} f(B, y) d y\right], \\
& E(A, B ; C):=\frac{1}{36}\left[\frac{(C-c)^{2}}{d-c}\left(\left|f_{x y}(a, c)\right| \frac{(A-a)^{2}}{b-a}+\left|f_{x y}(b, c)\right| \frac{(b-A)^{2}}{b-a}\right)\right. \\
&\left.\quad \frac{(d-C)^{2}}{d-c}\left(\left|f_{x y}(a, d)\right| \frac{(B-a)^{2}}{b-a}+\left|f_{x y}(b, d)\right| \frac{(b-B)^{2}}{b-a}\right)\right],
\end{aligned}
$$

and

$$
\begin{aligned}
R(A, B ; C):= & \frac{1}{18(b-a)}\left[\left|f_{x y}(A, c)\right| \frac{(C-c)^{2}}{d-c}\left((A-a)^{2}+(b-A)^{2}\right)\right. \\
& +\left|f_{x y}(a, C)\right|\left((A-a)^{2} \frac{(C-c)^{2}}{d-c}+(B-a)^{2} \frac{(d-C)^{2}}{d-c}\right) \\
& +2\left|f_{x y}(A, C)\right| \frac{(C-c)^{2}}{d-c}\left((A-a)^{2}+(b-A)^{2}\right) \\
& +2\left|f_{x y}(B, C)\right| \frac{(d-C)^{2}}{d-c}\left((B-a)^{2}+(b-B)^{2}\right) \\
& +\left|f_{x y}(b, C)\right|\left((b-A)^{2} \frac{(C-c)^{2}}{d-c}+(b-B)^{2} \frac{(d-C)^{2}}{d-c}\right) \\
& \left.+\left|f_{x y}(B, d)\right| \frac{(d-C)^{2}}{d-c}\left((B-a)^{2}+(b-B)^{2}\right)\right] .
\end{aligned}
$$

Proof. In order to show our results, we need to denote the following identity mappings:

$$
I(x)= \begin{cases}\frac{A-x}{A-a} a+\frac{x-a}{A-a} A, & \text { if } a \leq x \leq A \\ \frac{b-x}{b-A} A+\frac{x-A}{b-A} b, & \text { if } A \leq x \leq b \\ \frac{B-x}{B-a} a+\frac{x-a}{B-a} B, & \text { if } a \leq x \leq B \\ \frac{b-x}{b-B} B+\frac{x-B}{b-B} b, & \text { if } B \leq x \leq b\end{cases}
$$

and

$$
I(y)=\left\{\begin{array}{ll}
\frac{C-y}{C-c} c+\frac{y-c}{C-c} C, & \text { if } c \leq y \leq C \\
\frac{d-y}{d-C} C+\frac{y-C}{d-C} d, & \text { if } C \leq y \leq d
\end{array} .\right.
$$


Integration by parts, we have

$$
\begin{aligned}
& \frac{1}{(b-a)(d-c)} \int_{c}^{d} \int_{a}^{b} f(x, y) d x d y+M(A, B ; C)-N(A, B ; C) \\
= & \frac{1}{(b-a)(d-c)} \int_{c}^{d} \int_{a}^{b} w_{1}(x, y) \cdot f_{x y}(x, y) d x d y
\end{aligned}
$$

and then

$$
\begin{aligned}
& \left|\frac{1}{(b-a)(d-c)} \int_{c}^{d} \int_{a}^{b} f(x, y) d x d y+M(A, B ; C)-N(A, B ; C)\right| \\
\leq & \frac{1}{(b-a)(d-c)} \int_{c}^{d} \int_{a}^{b}\left|w_{1}(x, y)\right| \times\left|f_{x y}(x, y)\right| d x d y .
\end{aligned}
$$

By using the identity mappings (2.3) and (2.4), we have

$$
\begin{aligned}
& \int_{c}^{d} \int_{a}^{b}|w(x, y)| \times\left|f_{x y}(I(x), I(y))\right| d x d y \\
= & \int_{c}^{C} \int_{a}^{A}(x-a)(y-c)\left|f_{x y}\left(\frac{A-x}{A-a} a+\frac{x-a}{A-a} A, \frac{C-y}{C-c} c+\frac{y-c}{C-c} C\right)\right| d x d y \\
& +\int_{C}^{d} \int_{a}^{B}(x-a)(d-y)\left|f_{x y}\left(\frac{B-x}{B-a} a+\frac{x-a}{B-a} B, \frac{d-y}{d-C} C+\frac{y-C}{d-C} d\right)\right| d x d y \\
& +\int_{c}^{C} \int_{A}^{b}(b-x)(y-c)\left|f_{x y}\left(\frac{b-x}{b-A} A+\frac{x-A}{b-A} b, \frac{C-y}{C-c} c+\frac{y-c}{C-c} C\right)\right| d x d y \\
& +\int_{C}^{d} \int_{B}^{b}(b-x)(d-y)\left|f_{x y}\left(\frac{b-x}{b-B} B+\frac{x-B}{b-B} b, \frac{d-y}{d-C} C+\frac{y-C}{d-C} d\right)\right| d x d y \\
= & I_{1}+I_{2}+I_{3}+I_{4} .
\end{aligned}
$$

By using the convexity of $\left|f_{x y}\right|$ on the co-ordinates on $\Delta$ and the inequality (1.2) in $I_{1}, I_{2}, I_{3}$ and $I_{4}$, then we have

$$
\begin{aligned}
I_{1} \leq & \frac{1}{(A-a)(C-c)} \int_{c}^{C} \int_{a}^{A}(x-a)(y-c) \\
& \times\left[(A-x)(C-y)\left|f_{x y}(a, c)\right|+(A-x)(y-c)\left|f_{x y}(a, C)\right|\right. \\
& \left.+(x-a)(C-y)\left|f_{x y}(A, c)\right|+(x-a)(y-c)\left|f_{x y}(A, C)\right|\right] d x d y \\
I_{2} \leq & \frac{1}{(B-a)(d-C)} \int_{C}^{d} \int_{a}^{B}(x-a)(d-y) \\
& \times\left[(B-x)(d-y)\left|f_{x y}(a, C)\right|+(B-x)(y-C)\left|f_{x y}(a, d)\right|\right. \\
& \left.+(x-a)(d-y)\left|f_{x y}(B, C)\right|+(x-a)(y-C)\left|f_{x y}(B, d)\right|\right] d x d y
\end{aligned}
$$




$$
\begin{aligned}
I_{3} \leq & \frac{1}{(b-A)(C-c)} \int_{c}^{C} \int_{A}^{b}(b-x)(y-c) \\
& \times\left[(b-x)(C-y)\left|f_{x y}(A, c)\right|+(b-x)(y-c)\left|f_{x y}(A, C)\right|\right. \\
& \left.+(x-A)(C-y)\left|f_{x y}(b, c)\right|+(x-A)(y-c)\left|f_{x y}(b, C)\right|\right] d x d y \\
I_{4} \leq & \frac{1}{(b-B)(d-C)} \int_{C}^{d} \int_{B}^{b}(b-x)(d-y) \\
& \times\left[(b-x)(d-y)\left|f_{x y}(B, C)\right|+(b-x)(y-C)\left|f_{x y}(B, d)\right|\right. \\
& \left.+(x-B)(d-y)\left|f_{x y}(b, C)\right|+(x-B)(y-C)\left|f_{x y}(b, d)\right|\right] d x d y .
\end{aligned}
$$

By applying the identities (I), (II), (III) to the above four inequalities and then simplifying the results, we get the estimated bounds $E(A, B ; C)$ and $R(A, B ; C)$. The proof of the inequality $(2.2)$ is complete.

Corollary 1. Under the assumptions of Theorem 1 with $A=B=(a+b) / 2$ and $C=(c+d) / 2$, we have

$$
\begin{aligned}
& \left|\frac{1}{(b-a)(d-c)} \int_{c}^{d} \int_{a}^{b} f(x, y) d x d y+f\left(\frac{a+b}{2}, \frac{c+d}{2}\right)-P\right| \\
\leq & E\left(\frac{a+b}{2}, \frac{a+b}{2} ; \frac{c+d}{2}\right)+R\left(\frac{a+b}{2}, \frac{a+b}{2} ; \frac{c+d}{2}\right),
\end{aligned}
$$

where

$$
\begin{aligned}
& E\left(\frac{a+b}{2}, \frac{a+b}{2} ; \frac{c+d}{2}\right) \\
:= & \frac{(b-a)(d-c)}{144}\left[\frac{\left|f_{x y}(a, c)\right|+\left|f_{x y}(b, c)\right|+\left|f_{x y}(a, d)\right|+\left|f_{x y}(b, d)\right|}{4}\right], \\
& R\left(\frac{a+b}{2}, \frac{a+b}{2} ; \frac{c+d}{2}\right) \\
:= & \frac{(b-a)(d-c)}{144}\left[\left|f_{x y}\left(\frac{a+b}{2}, c\right)\right|+\left|f_{x y}\left(a, \frac{c+d}{2}\right)\right|\right. \\
& \left.+4\left|f_{x y}\left(\frac{a+b}{2}, \frac{c+d}{2}\right)\right|+\left|f_{x y}\left(b, \frac{c+d}{2}\right)\right|+\left|f_{x y}\left(\frac{a+b}{2}, d\right)\right|\right],
\end{aligned}
$$

and $P$ is as given in Theorem $B$.

The Corollary 1 shows that we get the new estimated bound of Theorem B. 
Remark 1. By using the convexity of $\left|f_{x y}\right|$ on the co-ordinates on $\Delta$, we have the inequality

$$
\begin{gathered}
\left|f_{x y}\left(a, \frac{c+d}{2}\right)\right|+\left|f_{x y}\left(b, \frac{c+d}{2}\right)\right|+\left|f_{x y}\left(\frac{a+b}{2}, c\right)\right| \\
+\left|f_{x y}\left(\frac{a+b}{2}, d\right)\right|+4\left|f_{x y}\left(\frac{a+b}{2}, \frac{c+d}{2}\right)\right| \\
\leq 2\left(\left|f_{x y}(a, c)\right|+\left|f_{x y}(b, c)\right|+\left|f_{x y}(a, d)\right|+\left|f_{x y}(b, d)\right|\right),
\end{gathered}
$$

and then

$$
\begin{aligned}
& E\left(\frac{a+b}{2}, \frac{a+b}{2} ; \frac{c+d}{2}\right)+R\left(\frac{a+b}{2}, \frac{a+b}{2} ; \frac{c+d}{2}\right) \\
\leq & \frac{\left|f_{x y}(a, c)\right|+\left|f_{x y}(b, c)\right|+\left|f_{x y}(a, d)\right|+\left|f_{x y}(b, d)\right|}{4} \times \frac{(b-a)(d-c)}{16} .
\end{aligned}
$$

Hence the inequality (2.7) improves Theorem B.

Theorem 2. Let $f$ be defined as Theorem 1. If $\left|f_{x y}\right|^{q}$ is convex on the co-ordinates on $\Delta$ and $p, q>1,1 / p+1 / q=1$, then the following inequality holds:

$$
\begin{aligned}
& \left|\frac{1}{(b-a)(d-c)} \int_{c}^{d} \int_{a}^{b} f(x, y) d x d y+M(A, B ; C)-N(A, B ; C)\right| \\
\leq & {\left[E_{1}(A, B ; C ; q)+R_{1}(A, B ; C ; q)\right]^{\frac{1}{q}} \times W_{1}(A, B ; C ; p)^{\frac{1}{p}} }
\end{aligned}
$$

where $M(A, B ; C), N(A, B ; C)$ are as given in Theorem 1,

$$
\begin{aligned}
& E_{1}(A, B ; C ; q) \\
& \qquad \begin{array}{l}
:=\frac{1}{4}\left[\left(\frac{C-c}{d-c}\right)\left(\left|f_{x y}(a, c)\right|^{q}\left(\frac{A-a}{b-a}\right)+\left|f_{x y}(b, c)\right|^{q}\left(\frac{b-A}{b-a}\right)\right)\right. \\
\left.\quad+\left(\frac{d-C}{d-c}\right)\left(\left|f_{x y}(a, d)\right|^{q}\left(\frac{B-a}{b-a}\right)+\left|f_{x y}(b, d)\right|^{q}\left(\frac{b-B}{b-a}\right)\right)\right]
\end{array}
\end{aligned}
$$

$R_{1}(A, B ; C ; q)$

$$
\begin{aligned}
:= & \frac{1}{4}\left\{\left(\left[\left|f_{x y}(A, c)\right|^{q}\left(\frac{C-c}{d-c}\right)+\left|f_{x y}(B, d)\right|^{q}\left(\frac{d-C}{d-c}\right)\right]\right)\right. \\
& +\left|f_{x y}(a, C)\right|^{q}\left[\left(\frac{A-a}{b-a}\right)\left(\frac{C-c}{d-c}\right)+\left(\frac{B-a}{b-a}\right)\left(\frac{d-C}{d-c}\right)\right] \\
& +\left|f_{x y}(A, C)\right|^{q}\left(\frac{C-c}{d-c}\right)+\left|f_{x y}(B, C)\right|^{q}\left(\frac{d-C}{d-c}\right) \\
& \left.+\left|f_{x y}(b, C)\right|^{q}\left[\left(\frac{b-A}{b-a}\right)\left(\frac{C-c}{d-c}\right)+\left(\frac{b-B}{b-a}\right)\left(\frac{d-C}{d-c}\right)\right]\right\}
\end{aligned}
$$


and

$$
\begin{aligned}
W_{1}(A, B ; C ; p) & \\
:= & \frac{1}{(p+1)^{2}}\left[\frac{(C-c)^{p+1}}{d-c}\left(\frac{(A-a)^{p+1}}{b-a}+\frac{(b-A)^{p+1}}{b-a}\right)\right. \\
& \left.+\frac{(d-C)^{p+1}}{d-c}\left(\frac{(B-a)^{p+1}}{b-a}+\frac{(b-B)^{p+1}}{b-a}\right)\right] .
\end{aligned}
$$

Proof. From the inequality (2.5) and Hölder's inequality, we have

$$
\begin{aligned}
& \left|\frac{1}{(b-a)(d-c)} \int_{c}^{d} \int_{a}^{b} f(x, y) d x d y+M(A, B ; C)-N(A, B ; C)\right| \\
\leq & \frac{1}{(b-a)(d-c)}\left(\int_{c}^{d} \int_{a}^{b}\left|w_{1}(x, y)\right|^{p} d x d y\right)^{\frac{1}{p}}\left(\int_{c}^{d} \int_{a}^{b}\left|f_{x y}(x, y)\right|^{q} d x d y\right)^{\frac{1}{q}} .
\end{aligned}
$$

By using the simple integration techniques, we have the identity

$$
\begin{aligned}
\int_{c}^{d} \int_{a}^{b}\left|w_{1}(x, y)\right|^{p} d x d y \\
=\frac{1}{(p+1)^{2}}\left[(C-c)^{p+1}\left((A-a)^{p+1}+(b-A)^{p+1}\right)\right. \\
\left.\quad+(d-C)^{p+1}\left((B-a)^{p+1}+(b-B)^{p+1}\right)\right]
\end{aligned}
$$

and $\int_{c}^{d} \int_{a}^{b}\left|w_{1}(x, y)\right|^{p} /[(b-a)(d-c)] d x d y$ is denoted by $W_{1}(A, B ; C ; p)$.

Now, by using the identities (2.3) and (2.4), and the convexity of $\left|f_{x y}\right|^{q}$ on the co-ordinates on $\Delta$, we have

$$
\begin{aligned}
& \int_{c}^{d} \int_{a}^{b}\left|f_{x y}(x, y)\right|^{q} d x d y \\
\leq & \frac{1}{(A-a)(C-c)} \int_{c}^{C} \int_{a}^{A}\left[(A-x)(C-y)\left|f_{x y}(a, c)\right|^{q}\right. \\
& +(A-x)(y-c)\left|f_{x y}(a, C)\right|^{q}+(x-a)(C-y)\left|f_{x y}(A, c)\right|^{q} \\
& \left.+(x-a)(y-c)\left|f_{x y}(A, C)\right|^{q}\right] d x d y \\
+ & \frac{1}{(b-A)(C-c)} \int_{c}^{C} \int_{A}^{b}\left[(b-x)(C-y)\left|f_{x y}(A, c)\right|^{q}\right. \\
& +(b-x)(y-c)\left|f_{x y}(A, C)\right|^{q}+(x-A)(C-y)\left|f_{x y}(b, c)\right|^{q} \\
& \left.+(x-A)(y-c)\left|f_{x y}(b, C)\right|^{q}\right] d x d y
\end{aligned}
$$




$$
\begin{aligned}
+ & \frac{1}{(B-a)(d-C)} \int_{C}^{d} \int_{a}^{B}\left[(B-x)(d-y)\left|f_{x y}(a, C)\right|^{q}\right. \\
& +(B-x)(y-C)\left|f_{x y}(a, d)\right|^{q}+(x-a)(d-y)\left|f_{x y}(B, C)\right|^{q} \\
& \left.+(x-a)(y-C)\left|f_{x y}(B, d)\right|^{q}\right] d x d y \\
+ & \frac{1}{(b-B)(d-C)} \int_{C}^{d} \int_{B}^{b}\left[(b-x)(d-y)\left|f_{x y}(B, C)\right|^{q}\right. \\
& +(b-x)(y-C)\left|f_{x y}(B, d)\right|^{q}+(x-B)(d-y)\left|f_{x y}(b, C)\right|^{q} \\
& \left.+(x-B)(y-C)\left|f_{x y}(b, d)\right|^{q}\right] d x d y
\end{aligned}
$$

We evaluate the integrals in the above inequality (2.9) and then simplify the integrals to obtain the estimated bounds $E_{1}(A, B ; C ; q)$ and $R_{1}(A, B ; C ; q)$. This completes the proof.

Corollary 2. Under the assumptions of Theorem 2 with $A=B=(a+b) / 2$ and $C=(c+d) / 2$, we have

$$
\begin{aligned}
& \left|\frac{1}{(b-a)(d-c)} \int_{c}^{d} \int_{a}^{b} f(x, y) d x d y+f\left(\frac{a+b}{2}, \frac{c+d}{2}\right)-P\right| \\
\leq & {\left[E_{1}\left(\frac{a+b}{2}, \frac{a+b}{2} ; \frac{c+d}{2} ; q\right)+R_{1}\left(\frac{a+b}{2}, \frac{a+b}{2} ; \frac{c+d}{2} ; q\right)\right]^{\frac{1}{q}} } \\
& \times W_{1}\left(\frac{a+b}{2}, \frac{a+b}{2} ; \frac{c+d}{2} ; p\right)^{\frac{1}{p}}
\end{aligned}
$$

where $P$ is as given in Theorem B,

$$
\begin{aligned}
& E_{1}\left(\frac{a+b}{2}, \frac{a+b}{2} ; \frac{c+d}{2} ; q\right) \\
:= & \frac{1}{4} \times \frac{\left|f_{x y}(a, c)\right|^{q}+\left|f_{x y}(b, c)\right|^{q}+\left|f_{x y}(a, d)\right|^{q}+\left|f_{x y}(b, d)\right|^{q}}{4}, \\
& R_{1}\left(\frac{a+b}{2}, \frac{a+b}{2} ; \frac{c+d}{2} ; q\right) \\
:= & \frac{1}{8}\left[\left|f_{x y}\left(\frac{a+b}{2}, c\right)\right|^{q}+\left|f_{x y}\left(a, \frac{c+d}{2}\right)\right|^{q}+\left|f_{x y}\left(b, \frac{c+d}{2}\right)\right|^{q}\right. \\
& \left.+2\left|f_{x y}\left(\frac{a+b}{2}, \frac{c+d}{2}\right)\right|^{q}+\left|f_{x y}\left(\frac{a+b}{2}, d\right)\right|^{q}\right]
\end{aligned}
$$

and

$$
W_{1}\left(\frac{a+b}{2}, \frac{a+b}{2} ; \frac{c+d}{2} ; p\right):=\left[\frac{(b-a)(d-c)}{4(p+1)^{2 / p}}\right]^{p}
$$


The Corollary 2 shows that we get the new estimated bound of Theorem C.

Remark 2. By using the convexity of $\left|f_{x y}\right|^{q}$ on the co-ordinates on $\Delta$, we have the inequality

$$
\begin{aligned}
& \left|f_{x y}\left(\frac{a+b}{2}, c\right)\right|^{q}+\left|f_{x y}\left(a, \frac{c+d}{2}\right)\right|^{q}+\left|f_{x y}\left(b, \frac{c+d}{2}\right)\right|^{q} \\
& +2\left|f_{x y}\left(\frac{a+b}{2}, \frac{c+d}{2}\right)\right|^{q}+\left|f_{x y}\left(\frac{a+b}{2}, d\right)\right|^{q} \\
\leq & \frac{3}{2}\left(\left|f_{x y}(a, c)\right|^{q}+\left|f_{x y}(b, c)\right|^{q}+\left|f_{x y}(a, d)\right|^{q}+\left|f_{x y}(b, d)\right|^{q}\right),
\end{aligned}
$$

and then

$$
\begin{aligned}
& E_{1}\left(\frac{a+b}{2}, \frac{a+b}{2} ; \frac{c+d}{2} ; q\right)+R_{1}\left(\frac{a+b}{2}, \frac{a+b}{2} ; \frac{c+d}{2} ; q\right) \\
\leq & \frac{\left|f_{x y}(a, c)\right|^{q}+\left|f_{x y}(b, c)\right|^{q}+\left|f_{x y}(a, d)\right|^{q}+\left|f_{x y}(b, d)\right|^{q}}{4} .
\end{aligned}
$$

Hence the inequality (2.10) improves Theorem C.

Theorem 3. Let $f$ be defined as Theorem 1. If $\left|f_{x y}\right|^{q}$ is convex on the co-ordinates on $\Delta$ and $q \geq 1$, then the following inequality holds:

$$
\begin{aligned}
& \left|\frac{1}{(b-a)(d-c)} \int_{c}^{d} \int_{a}^{b} f(x, y) d x d y+M(A, B ; C)-N(A, B ; C)\right| \\
\leq & \left(E_{2}(A, B ; C ; q)+R_{2}(A, B, C ; q)\right)^{\frac{1}{q}} \times W_{2}(A, B ; C)^{1-\frac{1}{q}}
\end{aligned}
$$

where

$$
\begin{aligned}
& E_{2}(A, B ; C ; q) \\
:= & \frac{1}{36}\left[\frac{(C-c)^{2}}{d-c}\left(\left|f_{x y}(a, c)\right|^{q} \frac{(A-a)^{2}}{b-a}+\left|f_{x y}(b, c)\right|^{q} \frac{(b-A)^{2}}{b-a}\right)\right. \\
& \left.+\frac{(d-C)^{2}}{d-c}\left(\left|f_{x y}(a, d)\right|^{q} \frac{(B-a)^{2}}{b-a}+\left|f_{x y}(b, d)\right|^{q} \frac{(b-B)^{2}}{b-a}\right)\right], \\
& R_{2}(A, B ; C ; q) \\
:= & \frac{1}{18(b-a)}\left[\left|f_{x y}(A, c)\right|^{q} \frac{(C-c)^{2}}{d-c}\left((A-a)^{2}+(b-A)^{2}\right)\right. \\
& +\left|f_{x y}(a, C)\right|^{q}\left((A-a)^{2} \frac{(C-c)^{2}}{d-c}+(B-a)^{2} \frac{(d-C)^{2}}{d-c}\right) \\
& +2\left|f_{x y}(A, C)\right|^{q} \frac{(C-c)^{2}}{d-c}\left((A-a)^{2}+(b-A)^{2}\right)
\end{aligned}
$$




$$
\begin{aligned}
& +2\left|f_{x y}(B, C)\right|^{q} \frac{(d-C)^{2}}{d-c}\left((B-a)^{2}+(b-B)^{2}\right) \\
& +\left|f_{x y}(b, C)\right|^{q}\left((b-A)^{2} \frac{(C-c)^{2}}{d-c}+(b-B)^{2} \frac{(d-C)^{2}}{d-c}\right) \\
& \left.+\left|f_{x y}(B, d)\right|^{q} \frac{(d-C)^{2}}{d-c}\left((B-a)^{2}+(b-B)^{2}\right)\right]
\end{aligned}
$$

and

$$
\begin{aligned}
W_{2}(A, B ; C):= & \frac{1}{4}\left[\frac{(C-c)^{2}}{d-c}\left(\frac{(A-a)^{2}}{b-a}+\frac{(b-A)^{2}}{b-a}\right)\right. \\
& \left.+\frac{(d-C)^{2}}{d-c}\left(\frac{(B-a)^{2}}{b-a}+\frac{(b-B)^{2}}{b-a}\right)\right] .
\end{aligned}
$$

Proof. By using the inequality (2.5) and the power mean inequality, we have

$$
\begin{aligned}
& \left|\frac{1}{(b-a)(d-c)} \int_{c}^{d} \int_{a}^{b} f(x, y) d x d y+M(A, B ; C)-N(A, B ; C)\right| \\
& \leq \frac{1}{(b-a)(d-c)}\left(\int_{c}^{d} \int_{a}^{b}\left|w_{1}(x, y)\right| d x d y\right)^{1-\frac{1}{q}} \\
& \left(\int_{c}^{d} \int_{a}^{b}\left|w_{1}(x, y)\right|\left|f_{x y}(x, y)\right|^{q} d x d y\right)^{\frac{1}{q}} .
\end{aligned}
$$

Now, by using the identities (2.4) and (2.5), and the convexity of $\left|f_{x y}\right|^{q}$ on the co-ordinates on $\Delta$, we have

$$
\begin{aligned}
& \int_{c}^{d} \int_{a}^{b}\left|w_{1}(x, y)\right|\left|f_{x y}(x, y)\right|^{q} d x d y \\
\leq & \frac{1}{(A-a)(C-c)} \int_{c}^{C} \int_{a}^{A}(x-a)(y-c) \\
& \times\left[(A-x)(C-y)\left|f_{x y}(a, c)\right|^{q}+(A-x)(y-c)\left|f_{x y}(a, C)\right|^{q}\right. \\
& \left.+(x-a)(C-y)\left|f_{x y}(A, c)\right|^{q}+(x-a)(y-c)\left|f_{x y}(A, C)\right|^{q}\right] d x d y \\
& +\frac{1}{(B-a)(d-C)} \int_{C}^{d} \int_{a}^{B}(x-a)(d-y) \\
& \times\left[(B-x)(d-y)\left|f_{x y}(a, C)\right|^{q}+(B-x)(y-C)\left|f_{x y}(a, d)\right|^{q}\right. \\
& \left.+(x-a)(d-y)\left|f_{x y}(B, C)\right|^{q}+(x-a)(y-C)\left|f_{x y}(B, d)\right|^{q}\right] d x d y \\
& +\frac{1}{(b-A)(C-c)^{2}} \int_{c}^{C} \int_{A}^{b}(b-x)(y-c) \\
& \times\left[(b-x)(C-y)\left|f_{x y}(A, c)\right|^{q}+(b-x)(y-c)\left|f_{x y}(A, C)\right|^{q}\right.
\end{aligned}
$$




$$
\begin{aligned}
& \left.+(x-A)(C-y)\left|f_{x y}(b, c)\right|^{q}+(x-A)(y-c)\left|f_{x y}(b, C)\right|^{q}\right] d x d y \\
& +\frac{1}{(b-B)(d-C)} \int_{C}^{d} \int_{B}^{b}(b-x)(d-y) \\
& \times\left[(b-x)(d-y)\left|f_{x y}(B, C)\right|^{q}+(b-x)(y-C)\left|f_{x y}(B, d)\right|^{q}\right. \\
& \left.+(x-B)(d-y)\left|f_{x y}(b, C)\right|^{q}+(x-B)(y-C)\left|f_{x y}(b, d)\right|^{q}\right] d x d y .
\end{aligned}
$$

Then we evaluate and simplify each integral in (2.12) by using the identities (I), (II), (III). In addition, we have

$$
\begin{aligned}
& \int_{c}^{d} \int_{a}^{b}|w(x, y)| d x d y \\
= & \frac{1}{4}\left[(C-c)^{2}\left((A-a)^{2}+(b-A)^{2}\right)+(d-C)^{2}\left((B-a)^{2}+(b-B)^{2}\right)\right],
\end{aligned}
$$

and $\int_{c}^{d} \int_{a}^{b}\left|w_{1}(x, y)\right| /[(b-a)(d-c)] d x d y$ is denoted by $W_{2}(A, B ; C)$. Then, we get the estimated bounds $E_{2}(A, B ; C ; q)$ and $R_{2}(A, B ; C ; q)$. This completes the proof.

Corollary 3. Under the assumptions of Theorem 3 with $A=B=(a+b) / 2$ and $C=(c+d) / 2$, we have

$$
\begin{aligned}
& \left|\frac{1}{(b-a)(d-c)} \int_{c}^{d} \int_{a}^{b} f(x, y) d x d y+f\left(\frac{a+b}{2}, \frac{c+d}{2}\right)-P\right| \\
\leq & {\left[E_{2}\left(\frac{a+b}{2}, \frac{a+b}{2} ; \frac{c+d}{2} ; q\right)+R_{2}\left(\frac{a+b}{2}, \frac{a+b}{2} ; \frac{c+d}{2} ; q\right)\right]^{\frac{1}{q}} } \\
& \times W_{2}\left(\frac{a+b}{2}, \frac{a+b}{2} ; \frac{c+d}{2}\right)^{1-\frac{1}{q}}
\end{aligned}
$$

where $P$ is as given in Theorem B,

$$
\begin{aligned}
& E_{2}\left(\frac{a+b}{2}, \frac{a+b}{2} ; \frac{c+d}{2} ; q\right) \\
:= & \frac{(b-a)(d-c)}{144} \times \frac{\left|f_{x y}(a, c)\right|^{q}+\left|f_{x y}(b, c)\right|^{q}+\left|f_{x y}(a, d)\right|^{q}+\left|f_{x y}(b, d)\right|^{q}}{4}, \\
& R_{2}\left(\frac{a+b}{2}, \frac{a+b}{2} ; \frac{c+d}{2} ; q\right) \\
:= & \frac{(b-a)(d-c)}{144}\left[\left|f_{x y}\left(\frac{a+b}{2}, c\right)\right|^{q}+\left|f_{x y}\left(a, \frac{c+d}{2}\right)\right|^{q}\right. \\
& \left.+4\left|f_{x y}\left(\frac{a+b}{2}, \frac{c+d}{2}\right)\right|^{q}+\left|f_{x y}\left(b, \frac{c+d}{2}\right)\right|^{q}+\left|f_{x y}\left(\frac{a+b}{2}, d\right)\right|^{q}\right]
\end{aligned}
$$


and

$$
W_{2}\left(\frac{a+b}{2}, \frac{a+b}{2} ; \frac{c+d}{2}\right):=\frac{(b-a)(d-c)}{16} .
$$

The Corollary 3 shows that we get the new estimated bound of Theorem D.

Remark 3. By using the convexity of $\left|f_{x y}\right|^{q}$ on the co-ordinates on $\Delta$, we have the inequality

$$
\begin{aligned}
& \left|f_{x y}\left(\frac{a+b}{2}, c\right)\right|^{q}+\left|f_{x y}\left(a, \frac{c+d}{2}\right)\right|^{q}+\left|f_{x y}\left(b, \frac{c+d}{2}\right)\right|^{q} \\
& +4\left|f_{x y}\left(\frac{a+b}{2}, \frac{c+d}{2}\right)\right|^{q}+\left|f_{x y}\left(\frac{a+b}{2}, d\right)\right|^{q} \\
\leq & 2\left(\left|f_{x y}(a, c)\right|^{q}+\left|f_{x y}(b, c)\right|^{q}+\left|f_{x y}(a, d)\right|^{q}+\left|f_{x y}(b, d)\right|^{q}\right),
\end{aligned}
$$

and then

$$
\begin{aligned}
& E\left(\frac{a+b}{2}, \frac{a+b}{2} ; \frac{c+d}{2} ; q\right)+R\left(\frac{a+b}{2}, \frac{a+b}{2} ; \frac{c+d}{2} ; q\right) \\
\leq & \frac{\left|f_{x y}(a, c)\right|^{q}+\left|f_{x y}(b, c)\right|^{q}+\left|f_{x y}(a, d)\right|^{q}+\left|f_{x y}(b, d)\right|^{q}}{4} \times \frac{(b-a)(d-c)}{16} .
\end{aligned}
$$

Hence the inequality (2.13) improves Theorem D.

Example 1. Let the function $f(x, y)$ be $x^{(q+2) / q} y^{(q+2) / q},(x, y) \in[0,1]^{2}$. Then the result of the right-hand side of $(1.6)$ is $(1 / 16)(1 / 4)^{1 / q}(1+(2 / q))^{2}$, whereas the right-hand side of $(2.13)$ is $(1 / 16)(1 / 9)^{1 / q}(1+(2 / q))^{2}$.

Theorem 4. Let $f$ be defined as Theorem 1. If $\left|f_{x y}\right|^{q}$ is concave on the co-ordinates on $\Delta$ and $1 \leq r \leq q$, then the following inequality holds:

$$
\begin{aligned}
& \left|\frac{1}{(b-a)(d-c)} \int_{c}^{d} \int_{a}^{b} f(x, y) d x d y+M_{1}\left(A_{1}, A_{2}, B ; C\right)-N_{1}\left(A_{1}, A_{2}, B ; C\right)\right| \\
& \quad \leq\left[E_{3}\left(A_{1}, A_{2}, B ; C ; r\right)+R_{3}\left(A_{1}, A_{2}, B ; C ; r\right)\right]^{\frac{1}{r}} \times W_{3}\left(A_{1}, A_{2}, B ; C ; r\right)^{\frac{r-1}{r}}
\end{aligned}
$$

where

$$
\begin{aligned}
M_{1}\left(A_{1}, A_{2}, B ; C\right):= & f\left(A_{1}, C\right) \frac{A_{1}-a}{b-a}+f(B, C) \frac{A_{2}-A_{1}}{b-a}+f\left(A_{2}, C\right) \frac{b-A_{2}}{b-a}, \\
N_{1}\left(A_{1}, A_{2}, B ; C\right):= & \frac{1}{b-a} \int_{a}^{b} f(x, C) d x+\frac{A_{1}-a}{b-a} \int_{c}^{d} \frac{f\left(A_{1}, y\right)}{d-c} d y \\
& +\frac{A_{2}-A_{1}}{b-a} \int_{c}^{d} \frac{f(B, y)}{d-c} d y+\frac{b-A_{2}}{b-a} \int_{c}^{d} \frac{f\left(A_{2}, y\right)}{d-c} d y,
\end{aligned}
$$




$$
\begin{aligned}
& E_{3}\left(A_{1}, A_{2}, B ; C ; r\right):=\frac{(C-c)^{2}}{4(d-c)} \\
& \quad \times\left(\frac{\left(A_{1}-a\right)^{2}}{b-a}\left|f_{x y}\left(\frac{a+2 A_{1}}{3}, \frac{c+2 C}{3}\right)\right|^{r}+\frac{\left(B-A_{1}\right)^{2}}{b-a}\left|f_{x y}\left(\frac{A_{1}+2 B}{3}, \frac{c+2 C}{3}\right)\right|^{r}\right. \\
& \left.\frac{\left(A_{1}-B\right)^{2}}{b-a}\left|f_{x y}\left(\frac{2 B+A_{2}}{3}, \frac{c+2 C}{3}\right)\right|^{r}+\frac{\left(b-A_{2}\right)^{2}}{b-a}\left|f_{x y}\left(\frac{2 A_{2}+b}{3}, \frac{c+2 C}{3}\right)\right|^{r}\right) \\
& R_{3}\left(A_{1}, A_{2}, B ; C ; r\right):=\frac{(d-C)^{2}}{4(d-c)} \\
& \quad \times\left(\frac{\left(A_{1}-a\right)^{2}}{b-a}\left|f_{x y}\left(\frac{a+2 A_{1}}{3}, \frac{2 C+d}{3}\right)\right|^{r}+\frac{\left(B-A_{1}\right)^{2}}{b-a}\left|f_{x y}\left(\frac{A_{1}+2 B}{3}, \frac{2 C+d}{3}\right)\right|^{r}\right. \\
& \left.\frac{\left(A_{1}-B\right)^{2}}{b-a}\left|f_{x y}\left(\frac{2 B+A_{2}}{3}, \frac{2 C+d}{3}\right)\right|^{r}+\frac{\left(b-A_{2}\right)^{2}}{b-a}\left|f_{x y}\left(\frac{2 A_{2}+b}{3}, \frac{2 C+d}{3}\right)\right|^{r}\right)
\end{aligned}
$$

and

$$
\begin{aligned}
W_{3}\left(A_{1}, A_{2}, B ; C ; r\right) & :=\frac{(C-c)^{2}+(d-C)^{2}}{d-c} \\
& \times \frac{\left(A_{1}-a\right)^{2}+\left(B-A_{1}\right)^{2}+\left(A_{2}-B\right)^{2}+\left(b-A_{2}\right)^{2}}{4(b-a)} .
\end{aligned}
$$

Proof. Let $w_{2}: \Delta \rightarrow \mathbb{R}$ be defined by

$$
w_{2}(x, y):= \begin{cases}(x-a)(y-c), & (x, y) \in\left[a, A_{1}\right] \times[c, C] \\ (x-a)(y-d), & (x, y) \in\left[a, A_{1}\right] \times[C, d] \\ \left(x-A_{1}\right)(y-c), & (x, y) \in\left[A_{1}, B\right] \times[c, C] \\ \left(x-A_{1}\right)(y-d), & (x, y) \in\left[A_{1}, B\right] \times[C, d] \\ \left(x-A_{2}\right)(y-c), & (x, y) \in\left[B, A_{2}\right] \times[c, C] \\ \left(x-A_{2}\right)(d-c), & (x, y) \in\left[B, A_{2}\right] \times[C, d] \\ (x-b)(y-c), & (x, y) \in\left[A_{2}, B\right] \times[c, C] \\ (x-b)(y-d), & (x, y) \in\left[A_{2}, B\right] \times[C, d]\end{cases}
$$

Integration by parts, we have

$$
\begin{gathered}
\frac{1}{(b-a)(d-c)} \int_{c}^{d} \int_{a}^{b} f(x, y) d x d y+\left(f\left(A_{1}, C\right) \frac{A_{1}-a}{b-a}\right. \\
\left.+f(B, C) \frac{A_{2}-A_{1}}{b-a}+f\left(A_{2}, C\right) \frac{b-A_{2}}{b-a}\right)-\left(\frac{1}{b-a} \int_{a}^{b} f(x, C) d x\right. \\
\left.+\frac{A_{1}-a}{b-a} \int_{c}^{d} \frac{f\left(A_{1}, y\right)}{d-c} d y+\frac{A_{2}-A_{1}}{b-a} \int_{c}^{d} \frac{f(B, y)}{d-c} d y+\frac{b-A_{2}}{b-a} \int_{c}^{d} \frac{f\left(A_{2}, y\right)}{d-c} d y\right) \\
=\frac{1}{(b-a)(d-c)} \int_{c}^{d} \int_{a}^{b} w_{2}(x, y) f_{x y}(x, y) d x d y .
\end{gathered}
$$


We denote $M_{1}\left(A_{1}, A_{2}, B ; C\right)$ and $N_{1}\left(A_{1}, A_{2}, B ; C\right)$ by

$$
M_{1}\left(A_{1}, A_{2}, B ; C\right):=f\left(A_{1}, C\right) \frac{A_{1}-a}{b-a}+f(B, C) \frac{A_{2}-A_{1}}{b-a}+f\left(A_{2}, C\right) \frac{b-A_{2}}{b-a}
$$

and

$$
\begin{aligned}
& N_{1}\left(A_{1}, A_{2}, B ; C\right):=\frac{1}{b-a} \int_{a}^{b} f(x, C) d x \\
& +\frac{A_{1}-a}{b-a} \int_{c}^{d} \frac{f\left(A_{1}, y\right)}{d-c} d y+\frac{A_{2}-A_{1}}{b-a} \int_{c}^{d} \frac{f(B, y)}{d-c} d y+\frac{b-A_{2}}{b-a} \int_{c}^{d} \frac{f\left(A_{2}, y\right)}{d-c} d y,
\end{aligned}
$$

respectively, and then

$$
\begin{aligned}
& \left|\frac{1}{(b-a)(d-c)} \int_{c}^{d} \int_{a}^{b} f(x, y) d x d y+M_{1}\left(A_{1}, A_{2}, B ; C\right)-N_{1}\left(A_{1}, A_{2}, B ; C\right)\right| \\
\leq & \frac{1}{(b-a)(d-c)} \int_{c}^{d} \int_{a}^{b}\left|w_{2}(x, y)\right| \times\left|f_{x y}(x, y)\right| d x d y .
\end{aligned}
$$

By Hölder's inequality, we have

$$
\begin{aligned}
& \int_{c}^{d} \int_{a}^{b}\left|w_{2}(x, y)\right| \times\left|f_{x y}(x, y)\right| d x d y \\
\leq & {\left[\int_{c}^{d} \int_{a}^{b}\left|w_{2}(x, y)\right| d x d y\right]^{(r-1) / r}\left[\int_{c}^{d} \int_{a}^{b}\left|w_{2}(x, y)\right|\left|f_{x y}(x, y)\right|^{r} d x d y\right]^{1 / r} . }
\end{aligned}
$$

Now, by using the simple integration techniques, we have

$$
\begin{aligned}
& \int_{c}^{d} \int_{a}^{b}\left|w_{2}(x, y)\right| d x d y \\
= & {\left[(C-c)^{2}+(d-C)^{2}\right] } \\
& \times \frac{\left(A_{1}-a\right)^{2}+\left(B-A_{1}\right)^{2}+\left(A_{2}-B\right)^{2}+\left(b-A_{2}\right)^{2}}{4},
\end{aligned}
$$

and $\int_{c}^{d} \int_{a}^{b}\left|w_{2}(x, y)\right| /[(b-a)(d-c)] d x d y$ is denoted by $W_{3}\left(A_{1}, A_{2}, B ; C ; r\right)$.

According to the definition of the $w_{2}(x, y)$, we get

$$
\begin{aligned}
& \int_{c}^{d} \int_{a}^{b}\left|w_{2}(x, y)\right|\left|f_{x y}(x, y)\right|^{r} d x d y \\
= & \int_{c}^{C} \int_{a}^{A_{1}}(x-a)(y-c)\left|f_{x y}(x, y)\right|^{r} d x d y \\
& +\int_{C}^{d} \int_{a}^{A_{1}}(x-a)(d-y)\left|f_{x y}(x, y)\right|^{r} d x d y
\end{aligned}
$$




$$
\begin{aligned}
& +\int_{c}^{C} \int_{A_{1}}^{B}\left(x-A_{1}\right)(y-c)\left|f_{x y}(x, y)\right|^{r} d x d y \\
& +\int_{C}^{d} \int_{A_{1}}^{B}\left(x-A_{1}\right)(d-y)\left|f_{x y}(x, y)\right|^{r} d x d y \\
& +\int_{c}^{C} \int_{B}^{A_{2}}\left(A_{2}-x\right)(y-c)\left|f_{x y}(x, y)\right|^{r} d x d y \\
& +\int_{C}^{d} \int_{B}^{A_{2}}\left(A_{2}-x\right)(d-y)\left|f_{x y}(x, y)\right|^{r} d x d y \\
& +\int_{c}^{C} \int_{A_{2}}^{b}(b-x)(y-c)\left|f_{x y}(x, y)\right|^{r} d x d y \\
& +\int_{C}^{d} \int_{A_{2}}^{b}(b-x)(d-y)\left|f_{x y}(x, y)\right|^{r} d x d y \\
& =J_{1}+J_{2}+J_{3}+J_{4}+J_{5}+J_{6}+J_{7}+J_{8} .
\end{aligned}
$$

We observe that $\left|f_{x y}\right|^{q}$ is concave, which implies $\left|f_{x y}\right|^{r}$ is concave for $r \in[1, q]$. Based on Jensen's integral inequality and $J_{1}-J_{8}$, we have

$$
\begin{aligned}
J_{1} & \leq \int_{a}^{A_{1}}(x-a)\left[\int_{c}^{C}(y-c) d y\right]\left|f_{x y}\left(x, c+\frac{\int_{c}^{C}(y-c)^{2} d y}{\int_{c}^{C}(y-c) d y}\right)\right|^{r} d x \\
& =\frac{1}{2}(C-c)^{2} \int_{a}^{A_{1}}(x-a)\left|f_{x y}\left(x, c+\frac{2}{3}(C-c)\right)\right|^{r} d x \\
& \leq \frac{1}{2}(C-c)^{2}\left[\int_{a}^{A_{1}}(x-a) d x\right]\left|f_{x y}\left(a+\frac{\int_{a}^{A_{1}}(x-a)^{2} d x}{\int_{a}^{A_{1}}(x-a) d x}, c+\frac{2}{3}(C-c)\right)\right|^{r} \\
& =\frac{1}{4}\left(A_{1}-a\right)^{2}(C-c)^{2}\left|f_{x y}\left(a+\frac{2}{3}\left(A_{1}-a\right), c+\frac{2}{3}(C-c)\right)\right|^{r} .
\end{aligned}
$$

In a similar way in $J_{1}$, we also have that

$$
\begin{aligned}
& J_{2} \leq \frac{1}{4}\left(A_{1}-a\right)^{2}(d-C)^{2}\left|f_{x y}\left(a+\frac{2}{3}\left(A_{1}-a\right), \frac{2}{3}(C-d)+d\right)\right|^{r}, \\
& J_{3} \leq \frac{1}{4}\left(B-A_{1}\right)^{2}(C-c)^{2}\left|f_{x y}\left(A_{1}+\frac{2}{3}\left(B-A_{1}\right), c+\frac{2}{3}(C-c)\right)\right|^{r}, \\
& J_{4} \leq \frac{1}{4}\left(B-A_{1}\right)^{2}(d-C)^{2}\left|f_{x y}\left(A_{1}+\frac{2}{3}\left(B-A_{1}\right), \frac{2}{3}(C-d)+d\right)\right|^{r}, \\
& J_{5} \leq \frac{1}{4}\left(A_{2}-B\right)^{2}(C-c)^{2}\left|f_{x y}\left(\frac{2}{3}\left(B-A_{2}\right)+A_{2}, c+\frac{2}{3}(C-c)\right)\right|^{r},
\end{aligned}
$$




$$
\begin{aligned}
& J_{6} \leq \frac{1}{4}\left(A_{2}-B\right)^{2}(d-C)^{2}\left|f_{x y}\left(\frac{2}{3}\left(B-A_{2}\right)+A_{2}, \frac{2}{3}(C-d)+d\right)\right|^{r} \\
& J_{7} \leq \frac{1}{4}\left(b-A_{2}\right)^{2}(C-c)^{2}\left|f_{x y}\left(\frac{2}{3}\left(A_{2}-b\right)+b, c+\frac{2}{3}(C-c)\right)\right|^{r}
\end{aligned}
$$

and

$$
J_{8} \leq \frac{1}{4}\left(b-A_{2}\right)^{2}(d-C)^{2}\left|f_{x y}\left(\frac{2}{3}\left(A_{2}-b\right)+b, c+\frac{2}{3}(C-c)\right)\right|^{r} .
$$

After simplifying the above inequalities, we have the bounds $E_{3}\left(A_{1}, A_{2}, B ; C ; r\right)$ and $R_{3}\left(A_{1}, A_{2}, B ; C ; r\right)$. This completes the proof.

Corollary 4. Under the assumptions of Theorem 4 with $A_{1}=B=A_{2}=$ $(a+b) / 2, C=(c+d) / 2$, we have

$$
\begin{aligned}
& \left|\frac{1}{(b-a)(d-c)} \int_{a}^{d} \int_{a}^{b} f(x, y) d x d y+f\left(\frac{a+b}{2}, \frac{c+d}{2}\right)-P\right| \\
\leq & \frac{(b-a)(d-c)}{16 \times 4^{1 / r}}\left[\left|f_{x y}\left(\frac{a+2 b}{3}, \frac{c+2 d}{3}\right)\right|^{r}+\left|f_{x y}\left(\frac{a+2 b}{3}, \frac{2 c+d}{3}\right)\right|^{r}\right. \\
& \left.+\left|f_{x y}\left(\frac{2 a+b}{3}, \frac{c+2 d}{3}\right)\right|^{r}+\left|f_{x y}\left(\frac{2 a+b}{3}, \frac{2 c+d}{3}\right)\right|^{r}\right]^{\frac{1}{r}},
\end{aligned}
$$

where $P$ is as given in Theorem $B$.

Remark 4. Under the assumptions of Corollary 4 with $r=1$. Then the inequality (2.15) reduces to the inequality $(1.7)$.

\section{Some Applications to Special Means}

As in [10] we shall consider extensions of arithmetic, logarithmic and generalized logarithmic means from positive real numbers. We take

$$
\begin{array}{lll}
A\left(\alpha_{1}, \alpha_{2}, \ldots, \alpha_{n}\right)=\frac{1}{n} \sum_{i=1}^{n} \alpha_{i}, & \alpha_{i} \in \mathbb{R}, & i=1,2, \ldots, n, \\
L(\alpha, \beta)=\frac{\beta-\alpha}{\ln |\beta|-\ln |\alpha|}, & |\alpha| \neq|\beta|, & \alpha \beta \neq 0, \\
L_{m}(\alpha, \beta)=\left[\frac{\beta^{m+1}-\alpha^{m+1}}{(m+1)(\beta-\alpha)}\right]^{\frac{1}{m}}, & m \in \mathbb{Z} \backslash\{-1,0\}, & \alpha, \beta \in 0, \alpha \neq \beta,
\end{array}
$$

where $\mathbb{Z}$ is the set of integers. 
Proposition 1. Let $a, b, c, d \in \mathbb{R}, a<b, c<d, 0 \notin[a, b], 0 \notin[c, d]$, and $m$, $n \in \mathbb{Z},|m| \geq 2$ and $|n| \geq 2$. Then, for $q \in[1, \infty)$, we have

$$
\begin{aligned}
& \left|\left(A(a, b)^{m}-L_{m}(a, b)^{m}\right)\left(A(c, d)^{n}-L_{n}(c, d)^{n}\right)\right| \leq \frac{|m||n|(b-a)(d-c)}{16} \\
& \times\left[A \left(\frac{|a|^{(m-1) q}|c|^{(n-1) q}}{4}, \frac{|b|^{(m-1) q}|c|^{(n-1) q}}{4}, \frac{|a|^{(m-1) q}|d|^{(n-1) q}}{4}, \frac{|b|^{(m-1) q}|d|^{(n-1) q}}{4},\right.\right. \\
& \left|\frac{a+b}{2}\right|^{(m-1) q}|c|^{(n-1) q},|a|^{(m-1) q}\left|\frac{c+d}{2}\right|^{(n-1) q},|b|^{(m-1) q}\left|\frac{c+d}{2}\right|^{(n-1) q}, \\
& \left.\left.4\left|\frac{a+b}{2}\right|^{(m-1) q}\left|\frac{c+d}{2}\right|^{(n-1) q},\left|\frac{a+b}{2}\right|^{(m-1) q}|d|^{(n-1) q}\right)\right]^{\frac{1}{q}},
\end{aligned}
$$

Proof. The proof is immediate from Corollary 3 with $f(x, y)=x^{m} y^{n}, x, y \in \mathbb{R}$, $m, n \in \mathbb{Z},|m| \geq 2,|n| \geq 2$.

Proposition 2. Suppose $a, b, c, d \in \mathbb{R}, a<b, c<d, 0 \notin[a, b], 0 \notin[c, d]$. Then, for $q \in[1, \infty)$, we have

$$
\begin{aligned}
& \left|\left(A(a, b)^{-1}-L^{-1}(a, b)\right)\left(A(c, d)^{-1}-L^{-1}(c, d)\right)\right| \leq \frac{(b-a)(d-c)}{16} \\
& \times\left[A \left(\left|\frac{a c}{4}\right|^{-2 q},\left|\frac{b c}{4}\right|^{-2 q},\left|\frac{a d}{4}\right|^{-2 q},\left|\frac{b d}{4}\right|^{-2 q}, 4\left|\frac{a c+a d+b c+b d}{4}\right|^{-2 q},\right.\right. \\
& \left.\left.\left|\frac{a c+b c}{2}\right|^{-2 q},\left|\frac{a c+a d}{2}\right|^{-2 q},\left|\frac{b c+b d}{2}\right|^{-2 q},\left|\frac{a d+b d}{2}\right|^{-2 q}\right)\right]^{\frac{1}{q}} .
\end{aligned}
$$

Proof. The result follows from Corollary 3 with $f(x, y)=1 /(x y)$.

Proposition 3. Let $a, b, c, d \in \mathbb{R}, a<b, c<d, 0 \notin[a, b], 0 \notin[c, d]$, and $m$, $n \in \mathbb{Z}, m, n \in[1,(1+r) / r)$. Then, for $r \in[1, q]$, we have

$$
\begin{aligned}
& \left|\left(A(a, b)^{m}-L_{m}(a, b)^{m}\right)\left(A(c, d)^{n}-L_{n}(c, d)^{n}\right)\right| \leq \frac{|m||n|(b-a)(d-c)}{16} \\
& \times A\left(\left|\frac{a+2 b}{3}\right|^{(m-1) r}\left|\frac{c+2 d}{3}\right|^{(n-1) r},\left|\frac{a+2 b}{3}\right|^{(m-1) r}\left|\frac{2 c+d}{3}\right|^{(n-1) r},\right. \\
& \left.\left|\frac{2 a+b}{3}\right|^{(m-1) r}\left|\frac{c+2 d}{3}\right|^{(n-1) r},\left|\frac{2 a+b}{3}\right|^{(m-1) r}\left|\frac{2 c+d}{3}\right|^{(n-1) r}\right)^{\frac{1}{r}} .
\end{aligned}
$$

Proof. The result follows from Corollary 4 with $f(x, y)=x^{m} y^{n}, x, y \in \mathbb{R}$, $m, n \in \mathbb{Z}, m, n \in[1,(1+r) / r)$. 


\section{Some Cubature Formulas}

Let $d_{1}$ be a partition $a=x_{0}<x_{1}<\ldots<x_{m-1}<x_{m}=b$ of the interval $[a, b]$ and $d_{2}$ be a partition $c=y_{0}<y_{1}<\ldots<y_{n-1}<y_{n}=d$ of the interval $[c, d]$. Now we consider the cubature formula

$$
\int_{c}^{d} \int_{a}^{b} f(x, y) d x d y=P\left(f, d_{1}, d_{2}\right)-T\left(f, d_{1}, d_{2}\right)+A_{i}\left(f, d_{1}, d_{2}\right), \quad i=1,2,
$$

where

$$
\begin{aligned}
P\left(f, d_{1}, d_{2}\right)= & \sum_{i=0}^{m-1} \sum_{j=0}^{n-1}\left[\int_{x_{i}}^{x_{i+1}} f\left(x, \frac{y_{j}+y_{j+1}}{2}\right) d x\right]\left(y_{j+1}-y_{j}\right) \\
& +\sum_{i=0}^{m-1} \sum_{j=0}^{n-1}\left[\int_{y_{j}}^{y_{j+1}} f\left(\frac{x_{i}+x_{i+1}}{2}, y\right) d y\right]\left(x_{i+1}-x_{i}\right)
\end{aligned}
$$

and

$$
T\left(f, d_{1}, d_{2}\right)=\sum_{i=0}^{m-1} \sum_{j=0}^{n-1} f\left(\frac{x_{i}+x_{i+1}}{2}, \frac{y_{j}+y_{j+1}}{2}\right)\left(x_{i+1}-x_{i}\right)\left(y_{j+1}-y_{j}\right) .
$$

Accordingly, $A_{i}\left(f, d_{1}, d_{2}\right)$ denotes the associated approximation error.

Proposition 4. If $\left|f_{x y}\right|^{q}$ is convex on the co-ordinates on $\Delta$ and $q \geq 1$, then for the partitions $d_{1}$ of $[a, b]$ and $d_{2}$ of $[c, d]$, the error satisfies

$$
\begin{aligned}
& A_{1}\left(f, d_{1}, d_{2}\right) \\
\leq & \sum_{i=0}^{m-1} \sum_{j=0}^{n-1} \frac{\left[\left(x_{i+1}-x_{i}\right)\left(y_{j+1}-y_{j}\right)\right]^{2-\frac{1}{q}}}{16^{1-\frac{1}{q}}}\left[E_{i}\left(f, d_{1}, d_{2}\right)+R_{i}\left(f, d_{1}, d_{2}\right)\right]^{\frac{1}{q}} \\
\leq & \frac{1}{16} \sum_{i=0}^{m-1} \sum_{j=0}^{n-1}\left(x_{i+1}-x_{i}\right)^{2}\left(y_{j+1}-y_{j}\right)^{2} \\
& \times\left[\frac{\left|f_{x y}\left(x_{i}, y_{j}\right)\right|^{q}+\left|f_{x y}\left(x_{i+1}, y_{j}\right)\right|^{q}+\left|f_{x y}\left(x_{i}, y_{j+1}\right)\right|^{q}+\left|f_{x y}\left(x_{i+1}, y_{j+1}\right)\right|^{q}}{4}\right]^{\frac{1}{q}} \\
\leq & \frac{1}{16} \sum_{i=0}^{m-1} \sum_{j=0}^{n-1}\left(x_{i+1}-x_{i}\right)^{2}\left(y_{j+1}-y_{j}\right)^{2} \\
& \times \max \left\{\left|f_{x y}(a, c)\right|,\left|f_{x y}(b, c)\right|,\left|f_{x y}(a, d)\right|,\left|f_{x y}(b, d)\right|\right\}
\end{aligned}
$$

where 


$$
\begin{aligned}
& E_{i}\left(f, d_{1}, d_{2}\right)=\frac{\left(x_{i+1}-x_{i}\right)\left(y_{j+1}-y_{j}\right)}{144} \\
& \times \frac{\left|f_{x y}\left(x_{i}, y_{j}\right)\right|^{q}+\left|f_{x y}\left(x_{i+1}, y_{j}\right)\right|^{q}+\left|f_{x y}\left(x_{i}, y_{j+1}\right)\right|^{q}+\left|f_{x y}\left(x_{i+1}, y_{j+1}\right)\right|^{q}}{4}
\end{aligned}
$$

and

$$
\begin{aligned}
R_{i}\left(f, d_{1}, d_{2}\right)= & \frac{\left(x_{i+1}-x_{i}\right)\left(y_{j+1}-y_{j}\right)}{144}\left[\left|f_{x y}\left(\frac{x_{i}+x_{i+1}}{2}, y_{j}\right)\right|^{q}\right. \\
& +4\left|f_{x y}\left(\frac{x_{i}+x_{i+1}}{2}, \frac{y_{j}+y_{j+1}}{2}\right)\right|^{q}+\left|f_{x y}\left(x_{i}, \frac{y_{j}+y_{j+1}}{2}\right)\right|^{q} \\
& +\left|f_{x y}\left(x_{i+1}, \frac{y_{j}+y_{j+1}}{2}\right)\right|^{q}+\left|f_{x y}\left(\frac{x_{i}+x_{i+1}}{2}, y_{j+1}\right)\right|^{q} .
\end{aligned}
$$

Proof. Apply Corollary 3 on the subintervals $\left[x_{i}, x_{i+1}\right](i=0,1,2 \ldots m-1)$ of the partition $d_{1}$ and $\left[y_{j}, y_{j+1}\right](j=0,1,2 \ldots n-1)$ of the partition $d_{2}$ to obtain

$$
\begin{aligned}
& \mid \int_{x_{i}}^{x_{i+1}} \int_{y_{j}}^{y_{j+1}} f(x, y) d y d x+f\left(\frac{x_{i}+x_{i+1}}{2}, \frac{y_{j}+y_{j+1}}{2}\right)\left(x_{i+1}-x_{i}\right)\left(y_{j+1}-y_{j}\right) \\
& -\left(y_{j+1}-y_{j}\right) \int_{x_{i}}^{x_{i+1}} f\left(x, \frac{y_{j}+y_{j+1}}{2}\right) d x-\left(x_{i+1}-x_{i}\right) \int_{y_{j}}^{y_{j+1}} f\left(\frac{x_{i}+x_{i+1}}{2}, y\right) d y \mid \\
& \leq \frac{\left(x_{i+1}-x_{i}\right)^{2-\frac{1}{q}}\left(y_{j+1}-y_{j}\right)^{2-\frac{1}{q}}}{16^{1-\frac{1}{q}}}\left[E_{i}\left(f, d_{1}, d_{2}\right)+R_{i}\left(f, d_{1}, d_{2}\right)\right]^{\frac{1}{q}} .
\end{aligned}
$$

Hence, we have

$$
\begin{aligned}
& \left|\int_{c}^{d} \int_{a}^{b} f(x, y) d x d y+T\left(f, d_{1}, d_{2}\right)-P\left(f, d_{1}, d_{2}\right)\right| \\
\leq & \sum_{i=0}^{m-1} \sum_{j=0}^{n-1} \mid \int_{x_{i}}^{x_{i+1}} \int_{y_{j}}^{y_{j+1}} f(x, y) d y d x \\
& +f\left(\frac{x_{i}+x_{i+1}}{2}, \frac{y_{j}+y_{j+1}}{2}\right)\left(x_{i+1}-x_{i}\right)\left(y_{j+1}-y_{j}\right) \\
& -\left(y_{j+1}-y_{j}\right) \int_{x_{i}}^{x_{i+1}} f\left(x, \frac{y_{j}+y_{j+1}}{2}\right) d x \\
& -\left(x_{i+1}-x_{i}\right) \int_{y_{j}}^{y_{j+1}} f\left(\frac{x_{i}+x_{i+1}}{2}, y\right) d y \mid \\
\leq & \sum_{i=0}^{m-1} \sum_{j=0}^{n-1} \frac{\left(x_{i+1}-x_{i}\right)^{2-\frac{1}{q}}\left(y_{j+1}-y_{j}\right)^{2-\frac{1}{q}}}{16^{1-\frac{1}{q}}}\left[E_{i}\left(f, d_{1}, d_{2}\right)+R_{i}\left(f, d_{1}, d_{2}\right)\right]^{\frac{1}{q}} \\
= & I .
\end{aligned}
$$


By using the convexity of $\left|f_{x y}\right|^{q}$ on the co-ordinates on $\Delta$, then we have

$$
\begin{aligned}
& E_{i}\left(f, d_{1}, d_{2}\right)+R_{i}\left(f, d_{1}, d_{2}\right) \leq \frac{\left(x_{i+1}-x_{i}\right)\left(y_{j+1}-y_{j}\right)}{16} \\
\times & \frac{\left|f_{x y}\left(x_{i}, y_{j}\right)\right|^{q}+\left|f_{x y}\left(x_{i+1}, y_{j}\right)\right|^{q}+\left|f_{x y}\left(x_{i}, y_{j+1}\right)\right|^{q}+\left|f_{x y}\left(x_{i+1}, y_{j+1}\right)\right|^{q}}{4} .
\end{aligned}
$$

The inequalities (4.1) and (4.2) imply that

$$
\begin{aligned}
I \leq & \frac{1}{16} \sum_{i=0}^{m-1} \sum_{j=0}^{n-1}\left(x_{i+1}-x_{i}\right)^{2}\left(y_{j+1}-y_{j}\right)^{2} \\
& \times\left[\frac{\left|f_{x y}\left(x_{i}, y_{j}\right)\right|^{q}+\left|f_{x y}\left(x_{i+1}, y_{j}\right)\right|^{q}+\left|f_{x y}\left(x_{i}, y_{j+1}\right)\right|^{q}+\left|f_{x y}\left(x_{i+1}, y_{j+1}\right)\right|^{q}}{4}\right]^{\frac{1}{q}} \\
\leq & \frac{1}{16} \sum_{i=0}^{m-1} \sum_{j=0}^{n-1}\left(x_{i+1}-x_{i}\right)^{2}\left(y_{j+1}-y_{j}\right)^{2} \\
& \times \max \left\{\left|f_{x y}\left(x_{i}, y_{j}\right)\right|,\left|f_{x y}\left(x_{i+1}, y_{j}\right)\right|,\left|f_{x y}\left(x_{i}, y_{j+1}\right)\right|,\left|f_{x y}\left(x_{i+1}, y_{j+1}\right)\right|\right\} \\
\leq & \frac{1}{16} \sum_{i=0}^{m-1} \sum_{j=0}^{n-1}\left(x_{i+1}-x_{i}\right)^{2}\left(y_{j+1}-y_{j}\right)^{2} \\
& \times \max \left\{\left|f_{x y}(a, c)\right|,\left|f_{x y}(b, c)\right|,\left|f_{x y}(a, d)\right|,\left|f_{x y}(b, d)\right|\right\} .
\end{aligned}
$$

The last inequality above follows from the convexity of the factor $\left|f_{x y}(x, y)\right|^{q}$.

Similarly, using Corollary 4, we can prove the following Proposition 5.

Proposition 5. If $\left|f_{x y}\right|^{q}$ is concave on the co-ordinates on $\Delta$ and $1 \leq r \leq q$, then for the partitions $d_{1}$ of $[a, b]$ and $d_{2}$ of $[c, d]$, the error satisfies

$$
\begin{aligned}
& A_{2}\left(f, d_{1}, d_{2}\right) \leq \sum_{i=0}^{m-1} \sum_{j=0}^{n-1} \frac{\left(x_{i+1}-x_{i}\right)\left(y_{j+1}-y_{j}\right)}{16 \times 4^{\frac{1}{r}}} \\
\times & {\left[\left|f_{x y}\left(\frac{x_{i}+2 x_{i+1}}{3}, \frac{y_{j}+2 y_{j+1}}{3}\right)\right|^{r}+\left|f_{x y}\left(\frac{x_{i}+2 x_{i+1}}{3}, \frac{2 y_{j}+y_{j+1}}{3}\right)\right|^{r}\right.} \\
+ & \left.\left|f_{x y}\left(\frac{2 x_{i}+x_{i+1}}{3}, \frac{y_{j}+2 y_{j+1}}{3}\right)\right|^{r}+\left|f_{x y}\left(\frac{2 x_{i}+x_{i+1}}{3}, \frac{2 y_{j}+y_{j+1}}{3}\right)\right|^{r}\right]^{\frac{1}{r}} \\
\leq & \sum_{i=0}^{m-1} \sum_{j=0}^{n-1} \frac{\left(x_{i+1}-x_{i}\right)\left(y_{j+1}-y_{j}\right)}{16}\left|f_{x y}\left(\frac{x_{i}+x_{i+1}}{2}, \frac{y_{j}+y_{j+1}}{2}\right)\right| .
\end{aligned}
$$




\section{REFERENCES}

1. S. S. Dragomir, Two mappings in connection to Hadamard's inequalities, J. Math. Anal. Appl., 167 (1992), 49-56.

2. S. S. Dragomir, On the Hadamard's inequality for convex on the co-ordinates in a rectangle from the plane, Taiwanese J. Math., 5(4) (2001), 775-788.

3. S. S. Dragomir and R. P. Agarwal, Two inequalities for differentiable mappings and applications to special means of real numbers and to trapezoidal formula, Appl. Math. Lett., 11(5) (1998), 91-95.

4. S. S. Dragomir and C. E. M. Pearce, Selected Topics on Hermite-Hadamard Inequalities and Applications, RGMIA Monographs, Victoria University, 2000, Online: http://www. staff.vu.edu.au/RGMIA/monographs/hermite_hadamard.html

5. J. Hadamard, Étude sur les propriétés des fonctions entières en particulier d'une fonction considérée par Riemann, J. Math. Pures Appl., 58 (1893), 171-215.

6. D.-Y. Hwang, K.-L. Tseng and K.-C. Hsu, Hadamard-type and Bullen-type inequalities for Lipschitzian functions and their applications, Comp. Math. Appl., 64 (2012), 651660 .

7. D.-Y. Hwang, K.-C. Hsu and K.-L. Tseng, Hadamard-type inequalities for Lipschitzian functions in one and two variables with applications, J. Math. Anal. Appl., 405 (2013), 546-554.

8. M. A. Latif and M. Alomari, Hadamard-type inequalities for product two convex functions on the co-ordinetes, Int Math Forum, 4(47) (2009), 2327-2338.

9. M. A. Latif and S. S. Dragomir, On some new inequalities for differentiable co-ordinated convex functions, J. of Inequal. and Appl., (2012), 2012:28, doi:10.1186/1029-242X2012-28.

10. C. E. M. Pearce and J. Pecarié, Inequalities for differentiable mappings with application to special means and quadrature formula. Appl. Math. Lett., 13 (2000), 51-55.

11. M. Z. Sarikaya, Erhan. Set, M. E. Ozdemir and S. S. Dragomir, New some Hadamard's type inequalities for co-ordinated convex functions, (2010), arXiv:1005.0700v1 [math. $\mathrm{CA}]$.

12. K.-L. Tseng, G.-S. Yang and K.-C. Hsu, On some inequalities of Hadamard's type and applications, Taiwanese J. Math., 13(6B) (2009), 1929-1948.

13. G.-S. Yang and K.-L. Tseng, On certain integral inequalities related to Hermite-Hadamard inequalities, J. Math. Anal. Appl., 239 (1999), 180-187.

14. G.-S. Yang and K.-L. Tseng, Inequalities of Hadamard's type for Lipschitzian mappings, J. Math. Anal. Appl., 260 (2001), 230-238.

15. G.-S. Yang and K.-L. Tseng, Inequalities of Hermite-Hadamard-Fejér type for convex functions and Lipschitzian functions, Taiwanese J. Math., 7(3) (2003), 433-440. 
16. G.-S. Yang, D.-Y. Hwang and K.-L. Tseng, Some inequalities for differentiable convex and concave mappings, Comp. Math. Appl., 47 (2004), 207-216.

\section{Kai-Chen Hsu}

Department of Applied Mathematics

Aletheia University

Tamsui, New Taipei City 25103

Taiwan

E-mail: victor8@mail.au.edu.tw

hsukaichen@gmail.com 